\title{
JOINT POWER CONTROL, MULTIUSER DETECTION AND BEAMFORMING FOR CDMA SYSTEMS
}

\author{
Aylin Yener \\ WINLAB, Rutgers University \\ yener@winlab.rutgers.edu
}

\author{
Roy D. Yates \\ WINLAB, Rutgers University \\ ryates@winlab.rutgers.edu
}

\author{
Sennur Ulukus \\ AT\&T Labs-Research \\ ulukus@research.att.com
}

\begin{abstract}
CDMA systems are limited by the interference that users create for each other. Several methods of controlling and/or suppressing the interference through power control, multiuser detection (temporal filtering) and beamforming (spatial filtering) are studied to increase the capacity of CDMA systems [1-3]. We investigate the capacity increase that is possible by combining power control with intelligent temporal and spatial receiver filter design. The SIR maximizing joint temporal-spatial receiver filters in unconstrained and constrained filter spaces are derived. Two-step iterative power control algorithms that converge to the optimum powers and the joint temporal and spatial receiver filters in the corresponding filter domains are given. A power control algorithm with less complex filter update procedure is given. It is observed that significant savings in total transmit power expended by all users in the system are possible if filtering in both domains are utilized compared with conventional power control and joint optimal power control and filtering in one domain.
\end{abstract}

\section{Introduction}

Code Division Multiple Access (CDMA) is a promising access scheme for fulfilling the ambitious goals of future wireless systems. It is well known that CDMA systems are interference limited and suffer from a phenomenon known as the near-far effect where strong users degrade the performance of the weak users significantly. In order to increase system capacity by controlling or suppressing the interference, three methods are commonly used: power control, multiuser detection and beamforming.

The aim of power control is to assign users with transmitter power levels so as to minimize the interference users create to each other while having a certain quality of service which is defined in terms of the Signal to Interference ratio (SIR) [1]. Traditional iterative power control approach assumes that one antenna and matched filter receivers are being used at the base stations and and each user employs an SIR based power update where the user's power is multiplied by the ratio of its target SIR to its current SIR. Thus, if the current SIR of the user is less than the target SIR then the power of that user is increased, otherwise it is decreased.
Multiuser detection [2] deals with designing advanced receivers to combat the near-far effect. A number of low complexity suboptimum receivers have been proposed following the development of the optimum detector [4]. Among these, the Minimum Mean Squared Error (MMSE) detector [5] minimizes the expected squared error between the transmitted signal and output of the receiver filter. It is also the linear filter which maximizes the output SIR.

Increasing the capacity of CDMA systems by employing antenna arrays at the base station has been proposed in [3]. The idea is to combine the outputs of multiple antenna array elements to make bit decisions for the user. This work assumes matched filter receivers in the time domain for each user as well as combining the array observations via a filter that is matched to the array response of the user.

Combining power control and multiuser detection for CDMA to find the jointly optimum powers and linear receiver filters was studied in [6]. It was shown that a distributed and iterative power control algorithm where each user optimizes its linear receiver filter before each power control update converged to the point where all users expend minimum transmit power and use the corresponding MMSE linear filters. A similar development arose in joint power control and beamforming for wireless networks in [7] where it was shown that a capacity increase is possible with power control if array observations are combined in the MMSE sense.

In this work, we combine the three approaches to further increase the capacity of a CDMA system by using power control, linear receivers and linear beamformers jointly. The aim is to assign each user with just enough transmit power and find the best way to process the received signal such that each user achieves its target SIR. For each user, we first find the joint optimal temporal and spatial filter that minimizes the mean squared error between the information bit and the decision statistic to be used to decode the user's bit assuming no constraints on the filter space. We find the iterative power control algorithm that updates the joint filters and the powers of all users that converges to the joint optimal powers and joint MMSE temporal-spatial filters. We then investigate spatial and temporal filters that are less complex to implement. We constrain the filter space and find the corresponding optimal temporal-spatial filters in this constrained space. We devise 
power control algorithms that use the resulting filters and converge to optimum powers and filters. It is observed that combining the three approaches, i.e. power control and intelligent combining in both spatial and temporal domains, leads to significant savings in total transmit power usage and can convert some highly loaded infeasible systems into feasible ones, increasing system capacity.

The structure of the paper is self explanatory, however we must note that space limitations preclude the derivation details and proofs.

\section{System Model}

We consider a multicell DS-CDMA system where each user is assigned a unique signature sequence and base station assignment has been done for all users. We assume a synchronous system with processing gain $G$. At each base station an antenna array of $K$ elements is employed. Thus the received signal at the output of the antenna array at the base station that user $i$ is assigned to is:

$$
\mathbf{r}_{i}(t)=\sum_{j=1}^{N} \sqrt{p_{j} h_{i j}} b_{j}(t) s_{j}(t) \mathbf{a}_{i j}+\mathbf{n}_{i}(t)
$$

where $p_{j}, b_{j}(t)$ and $s_{j}(t)$ are the transmit power, bit and the signature of user $j$, respectively. The uplink gain of user $j$ to the assigned base station of $i$ is $h_{i j}$ and $\mathbf{a}_{i j}$ is the array response vector of user $j$ (spatial signature) at the base station of $i$. Chip matched filtering the received signal and sampling at the chip rate, we have $G$ observations at the output of each of the $K$ antenna elements. The observations that will be used to decode the bit of user $i$ can be arranged as in the $G \times K$ matrix

$$
\mathbf{R}_{i}=\sum_{j=1}^{N} \sqrt{p_{j} h_{i j}} b_{j} \mathbf{s}_{j} \mathbf{a}_{i j}^{T}+\mathbf{N}_{i}
$$

where $k^{\text {th }}$ column of $\mathbf{R}_{i}$ represents the chip sampled outputs at the output of the $k^{\text {th }}$ antenna array element. $\mathbf{N}_{i}$ is the matrix that represents the spatially and temporally white noise, i.e. $E\left[\left[N_{i}\right]_{k l}\left[N_{i}\right]_{m n}\right]=\sigma^{2} \delta_{k m} \delta_{l n}$.

\section{Optimum Temporal-Spatial Filter- ing (OTSF)}

The detection of the information bits of the desired user is done by taking the sign of the decision statistic which is to be found using the observation matrix $\mathbf{R}_{i}$. Observations over the spatial and temporal domains are to be combined intelligently in making the bit decisions of the desired user. Our aim is to find a two dimensional linear filter, $\mathbf{X}_{i}$, that yields decision statistic $y_{i}=\sum_{j=1}^{G} \sum_{l=1}^{K}\left[X_{i}\right]_{j l}\left[R_{i}\right]_{j l}$. In particular, we aim for a filter that yields the minimum mean squared error (MMSE) between $y_{i}$ and $b_{i}$, i.e. we want to find a matrix filter $\mathbf{X}_{i}^{*}$ such that

$$
\begin{aligned}
\mathbf{X}_{i}^{*} & =\underset{\mathbf{X}}{\arg \min _{\mathbf{n}} E}\left[\left(\sum_{j=1}^{G} \sum_{l=1}^{K} X_{j l} R_{i, j l}-b_{i}\right)^{2}\right] \\
& =\arg \min _{\mathbf{X}} E\left[\left(\operatorname{tr}\left(\mathbf{X}^{T} \mathbf{R}_{i}\right)-b_{i}\right)^{2}\right]
\end{aligned}
$$

where $\operatorname{tr}(\cdot)$ is the trace operation.

The optimization problem (3) can be converted to an optimization problem with vector variables for easier manipulation [8]. Let $\mathbf{r}_{i}$ be the long vector obtained by stacking the columns of the received signal matrix, $\mathbf{R}_{i}$. The MMSE problem then can be reformulated as follows:

$$
\mathbf{x}_{i}^{*}=\arg \min _{\mathbf{x}} E\left[\left(\mathbf{x}^{T} \mathbf{r}_{i}-b_{i}\right)^{2}\right]
$$

Let us define $\mathbf{q}_{i j}$ as the combined temporal-spatial signature of user $j$ at the base station of user $i$. It is constructed by stacking columns of $\mathbf{s}_{j} \mathbf{a}_{i j}^{T}$ as a long vector of size $K G$. Then, the solution to the optimization problem (4) is given as [5]:

$$
\mathbf{x}_{i}^{*}=k_{i}\left(\sum_{j \neq i} p_{j} h_{i j} \mathbf{q}_{i j} \mathbf{q}_{i j}^{T}+\sigma^{2} \mathbf{I}\right)^{-1} \mathbf{q}_{i i}
$$

where

$$
k_{i}=\frac{\sqrt{p_{i} h_{i i}}}{1+p_{i} h_{i i} \mathbf{q}_{i i}^{T}\left(\sum_{j \neq i} \mathbf{q}_{i j} \mathbf{q}_{i j}^{T}+\sigma^{2} \mathbf{I}\right)^{-1} \mathbf{q}_{i i}}
$$

Note that the matrix $\sum_{j \neq i} \mathbf{q}_{i j} \mathbf{q}_{i j}^{T}+\sigma^{2} \mathbf{I}$ is necessarily positive definite (and thus has an inverse) for all $\sigma^{2}>0$. Note also that $\mathbf{X}_{i}^{*}$ then can be constructed by taking every $G$ element of $\mathbf{X}_{i}^{*}$ and putting as a column to $\mathbf{X}_{i}^{*}$.

\section{Power Control and OTSF}

In the previous section, we derived the joint MMSE filter for a CDMA system that employs spatial (through beamforming) and temporal processing (through multiuser detection) at the receiver. Our aim, in this section, is to find optimal powers, $p_{i}$, and matrix filters, $\mathbf{X}_{i}$ (or equivalently $\mathbf{x}_{i}$ ) for $i=1, \ldots, N$, such that the total transmitter power is minimized while each user $i$ satisfies its quality of service requirement, $\operatorname{SIR}_{i} \geq \gamma_{i}^{*}$, where $\gamma_{i}^{*}$, called the target SIR, is the minimum acceptable level of SIR for user $i$. The SIR of user $i$ at the output of the joint spatial and temporal filter can be expressed as

$$
\begin{aligned}
\operatorname{SIR}_{i} & =\frac{p_{i} h_{i i} \operatorname{tr}^{2}\left(\mathbf{X}_{i}^{\top} \mathbf{s}_{i} \mathbf{a}_{i i}^{\top}\right)}{\sum_{j \neq i} p_{j} h_{i j} \operatorname{tr}^{2}\left(\mathbf{X}_{i}^{\top} \mathbf{s}_{j} \mathbf{a}_{i j}^{\top}\right)+\sigma^{2}\left(\operatorname{tr}\left(\mathbf{X}_{i}^{\top} \mathbf{X}_{i}\right)\right)} \\
& =\frac{p_{i} h_{i i}\left(\mathbf{x}_{i}^{\top} \mathbf{q}_{i i}\right)^{2}}{\sum_{j \neq i} p_{j} h_{i j}\left(\mathbf{x}_{i}^{\top} \mathbf{q}_{i j}\right)^{2}+\sigma^{2}\left(\mathbf{x}_{i}^{\top} \mathbf{x}_{i}\right)}
\end{aligned}
$$


We can then state the optimization problem as

$$
\begin{array}{ll}
\min & \sum_{i=1}^{N} p_{i} \\
\text { s.t. } & p_{i} \geq \frac{\gamma_{i}^{*}}{h_{i i}} \frac{\sum_{j \neq i} p_{j} h_{i j}\left(\mathbf{x}_{i}^{\top} \mathbf{q}_{i j}\right)^{2}+\sigma^{2} \mathbf{x}_{i}^{\top} \mathbf{x}_{i}}{\left(\mathbf{x}_{i}^{\top} \mathbf{q}_{i i}\right)^{2}} \\
& p_{i} \geq 0, \quad \mathbf{x}_{i} \in \mathbf{R}^{K G} i=1, \ldots, N
\end{array}
$$

Note that in this formulation, we have used the long vector notation given in Section III. This is done to show the equivalence between (8) and the optimization problem studied in [6]. As in the case for the joint power control and temporal filtering, we can write (8) as

$$
\begin{array}{ll}
\min _{\left\{p_{i}\right\}} & \sum_{i=1}^{N} p_{i} \\
\text { s.t. } & p_{i} \geq \frac{\gamma_{i}^{*}}{h_{i i}} \min _{\mathbf{x}_{i}} \frac{\sum_{j \neq i} p_{j} h_{i j}\left(\mathbf{x}_{i}^{\top} \mathbf{q}_{i j}\right)^{2}+\sigma^{2} \mathbf{x}_{i}^{\top} \mathbf{x}_{i}}{\left(\mathbf{x}_{i}^{\top} \mathbf{q}_{i i}\right)^{2}} \\
& p_{i} \geq 0 \quad i=1, \ldots, N
\end{array}
$$

The minimization over $\mathbf{x}_{i}$, on the right hand side of each of the power constraints above, is equivalent to maximizing $\operatorname{SIR}_{i}$ given by (7) for a fixed power $p_{i}$. The solution is of the form of (5) scaled by any positive number [6].

Iterative power control algorithms of the form

$$
\mathbf{p}(n+1)=\mathbf{I}(\mathbf{p}(n))
$$

are shown to converge to the optimum power vector for standard interference functions $\mathbf{I}(\mathbf{p})$ [1]. Defining

$$
I_{i}(\mathbf{p})=\frac{\gamma_{i}^{*}}{h_{i i}} \min _{\mathbf{x}_{i}} \frac{\sum_{j \neq i} p_{j} h_{i j}\left(\mathbf{x}_{i}^{\top} \mathbf{q}_{i j}\right)^{2}+\sigma^{2}\left(\mathbf{x}_{i}^{\top} \mathbf{x}_{i}\right)}{\left(\mathbf{x}_{i}^{\top} \mathbf{q}_{i i}\right)^{2}}
$$

one can prove that the interference function $\mathbf{I}(\mathbf{p})$ whose $i^{\text {th }}$ component is given by (11) satisfies all three properties established in [1] and thus is standard [6]. This means that the power control iteration of the form of (10) converges to the optimum power vector. The implementation of the two step iterative power control algorithm is now given. The filter and power updates for user $i$ at iteration $n+1$ are given by

$$
\begin{aligned}
\hat{\mathbf{x}}_{i} & =k_{i}(n)\left(\sum_{j \neq i} p_{j}(n) h_{i j} \mathbf{q}_{i j} \mathbf{q}_{i j}^{\top}+\sigma^{2} \mathbf{I}\right)^{-1} \mathbf{q}_{i i} \\
p_{i}(n+1) & =\frac{\gamma_{i}^{*}\left(\sum_{j \neq i} p_{j}(n) h_{i j}\left(\hat{\mathbf{x}}_{i}^{\top} \mathbf{q}_{i j}\right)^{2}+\sigma^{2}\left|\hat{\mathbf{x}}_{i}\right|^{2}\right)}{h_{i i}\left(\hat{\mathbf{x}}_{i}^{\top} \mathbf{q}_{i i}\right)^{2}}
\end{aligned}
$$

where $|\cdot|$ denotes the norm of a vector and $k_{i}(n)$ is the scaling factor. Notice that any positive scalar multiple of $\hat{\mathbf{x}}_{i}$ yields the same power update in (13), so, calculation of $k_{i}(n)$ is actually not needed. If the SIR targets are feasible, then starting from any initial power vector and filter coefficients, the above algorithm converges to the unique componentwise minimum power fixed point with best possible joint spatial temporal filters.

\section{Constrained Temporal-Spatial Fil- tering (CTSF)}

Each filter update given by (12) needs a $K G \times K G$ matrix to be inverted and this has to be done before each power update. As this procedure may be computationally costly for large $K G$, one might want to consider less complex filtering procedures that nevertheless present capacity improvements for the system.

To this end, we consider a constrained class of rank 1 matrix filters: $\tilde{\mathbf{X}}_{i}=\mathbf{c}_{i} \mathbf{w}_{i}^{\top}$. Physically, the scheme is to combine the chip matched filter outputs using a linear filter at the output of each of the antenna (or equivalently linearly combining the all antenna array observations for each chip) followed by a linear combination of the resulting statistics. In this case, the optimization problem in (3) becomes

$$
\begin{aligned}
{\left[\mathbf{c}_{i}^{*}, \mathbf{w}_{i}^{*}\right] } & =\arg \min _{\mathbf{c}, \mathbf{w}} E\left[\left(\operatorname{tr}\left(\mathbf{w}_{i} \mathbf{c}_{i}^{\top} \mathbf{R}_{i}\right)-b_{i}\right)^{2}\right] \\
& =\arg \min _{\mathbf{c}_{i}, \mathbf{w}_{i}} E\left[\left(\mathbf{c}_{i}^{\top} \mathbf{R}_{i} \mathbf{w}_{i}-b_{i}\right)^{2}\right]
\end{aligned}
$$

Note that the resulting $\left[\mathbf{c}_{i}^{*}, \mathbf{w}_{i}^{*}\right]$ pair yields a matrix filter, $\mathbf{X}_{i}^{*}=$ $\mathbf{c}_{i}^{*} \mathbf{w}_{i}^{* \top}$, that is suboptimal for the optimization problem given in (3) since it is found in a constrained $\mathbf{X}$ space.

The MSE function in (14) can be expressed as

$$
\begin{aligned}
\mathrm{MSE}= & \sum_{j=1}^{N} p_{j} h_{i j}\left(\mathbf{c}_{i}^{\top} \mathbf{s}_{j}\right)^{2}\left(\mathbf{w}_{i}^{\top} \mathbf{a}_{i j}\right)^{2}+\sigma^{2}\left(\mathbf{c}_{i}^{\top} \mathbf{c}_{i}\right)\left(\mathbf{w}_{i}^{\top} \mathbf{w}_{i}\right) \\
& -2 \sqrt{p_{i} h_{i i}}\left(\mathbf{c}_{i}^{\top} \mathbf{s}_{i}\right)\left(\mathbf{w}_{i}^{\top} \mathbf{a}_{i i}\right)+1
\end{aligned}
$$

It can be shown that, although (15) is convex in $\mathbf{c}_{i}$ and $\mathbf{w}_{i}$, it is not jointly convex in both vector variables and the minimizer of MSE does not have a closed form expression. An iterative algorithm whose fixed point achieves the minimum MSE is devised as follows. Consider fixing the value of one of the filters, say $\tilde{\mathbf{w}}$. It is then possible to find the filter, $\overline{\mathbf{c}}$, that maximally decreases the MSE function in (15). The solution is the MMSE detector described in [5] where user $j$ 's received power is modified such that it is $p_{j} h_{i j}\left(\tilde{\mathbf{w}}^{\top} \mathbf{a}_{i j}\right)^{2}$. With some abuse of notation, we will call this filter $\overline{\mathbf{c}}=\operatorname{MMSE}(\tilde{\mathbf{w}})$ :

$$
\begin{aligned}
\overline{\mathbf{c}} & =\operatorname{MMSE}(\tilde{\mathbf{w}}) \\
& =\tilde{k}_{i}\left(\sum_{j \neq i} p_{j} h_{i j}\left(\tilde{\mathbf{w}}^{\top} \mathbf{a}_{i j}\right)^{2} \mathbf{s}_{j} \mathbf{s}_{j}^{T}+\sigma^{2}\left(\tilde{\mathbf{w}}^{\top} \tilde{\mathbf{w}}\right) \mathbf{I}\right)^{-1} \mathbf{s}_{i}
\end{aligned}
$$

The same argument can be made for the case where $\mathbf{c}_{i}$ is fixed to $\tilde{\mathbf{c}}$ and the spatial filter is found to maximally decrease the $\operatorname{MSE}, \overline{\mathbf{w}}=\operatorname{MMSE}(\tilde{\mathbf{c}})$ :

$$
\begin{aligned}
\overline{\mathbf{w}} & =\operatorname{MMSE}(\tilde{\mathbf{c}}) \\
& =\hat{k}_{i}\left(\sum_{j \neq i} p_{j} h_{i j}\left(\tilde{\mathbf{c}}^{\top} \mathbf{s}_{j}\right)^{2} \mathbf{a}_{i j} \mathbf{a}_{i j}^{T}+\sigma^{2}\left(\tilde{\mathbf{c}}^{\top} \tilde{\mathbf{c}}\right) \mathbf{I}\right)^{-1} \mathbf{a}_{i i}
\end{aligned}
$$

where $\tilde{k}_{i}$ and $\hat{k}_{i}$ are the appropriate scaling factors. Now, consider the following algorithm. Starting with the filter pair $\mathbf{c}(0), \mathbf{w}(0)$ and keeping $\mathbf{c}(0)$ fixed, one can find $\mathbf{w}(1)=$ $\operatorname{MMSE}(\mathbf{c}(0))$. Then keeping $\mathbf{w}(1)$ fixed, one can find $\mathbf{c}(1)=$ $\operatorname{MMSE}(\mathbf{w}(1))$ that further decreases the MSE in (15). It can 
be shown that the algorithm decreases the MSE monotonically, is convergent and the convergence point achieves the minimum MSE over all filters $\tilde{\mathbf{X}}_{i}=\mathbf{c}_{i} \mathbf{w}_{i}^{\top}$. It can also be shown that the algorithm increases $S_{i} R_{i}$ monotonically at each iteration converging to the maximum SIR. Iteration $n+1$ of this two step iterative algorithm for user $i$ is given below.

$$
\begin{aligned}
\mathbf{c}_{i}(n+1) & =\operatorname{MMSE}\left(\mathbf{w}_{i}(n)\right) \\
\mathbf{w}_{i}(n+1) & =\operatorname{MMSE}\left(\mathbf{c}_{i}(n+1)\right)
\end{aligned}
$$

Note that the above algorithm and the algorithm where $\mathbf{w}_{i}$ vector is updated before $\mathbf{c}_{i}$ have the same fixed point.

\section{Power Control and CTSF}

Now, consider the optimization problem where constrained temporal-spatial filters described in Section V are employed and the total transmit power expended by all active users is to be minimized while each user meets its quality of service requirement. The minimization problem can be expressed as

$$
\begin{array}{ll}
\min & \sum_{i=1}^{N} p_{i} \\
\text { s.t. } & p_{i} \geq \frac{\gamma_{i}^{*}}{h_{i i}} \frac{\sum_{j \neq i} p_{j} h_{i j}\left(\mathbf{c}_{i}^{\top} \mathbf{s}_{j}\right)^{2}\left(\mathbf{w}_{i}^{\top} \mathbf{a}_{i j}\right)^{2}+\sigma^{2}\left|\mathbf{c}_{i}\right|^{2}\left|\mathbf{w}_{i}\right|^{2}}{\left(\mathbf{c}_{i}^{\top} \mathbf{s}_{i}\right)^{2}\left(\mathbf{w}_{i}^{\top} \mathbf{a}_{i i}\right)^{2}} \\
& p_{i} \geq 0 \\
& \mathbf{c}_{i} \in \mathbf{R}^{G}, \mathbf{w}_{i} \in \mathbf{R}^{K} \quad i=1, \ldots, N
\end{array}
$$

Similar to going from (8) to (9), the minimization over $\mathbf{c}_{i}$ and $\mathbf{w}_{i}$ for each user $i$ can be moved to the constraint set. In that case, the SIR constraint for user $i$ becomes

$$
p_{i} \geq \frac{\gamma_{i}^{*}}{h_{i i}} J_{i}(\mathbf{p})
$$

where

$$
J_{i}(\mathbf{p})=\min _{\mathbf{c}_{i}, \mathbf{w}_{i}} \frac{\sum_{j \neq i} p_{j} h_{i j}\left(\mathbf{c}_{i}^{\top} \mathbf{s}_{j}\right)^{2}\left(\mathbf{w}_{i}^{\top} \mathbf{a}_{i j}\right)^{2}+\sigma^{2}\left|\mathbf{c}_{i}\right|^{2}\left|\mathbf{w}_{i}\right|^{2}}{\left(\mathbf{c}_{i}^{\top} \mathbf{s}_{i}\right)^{2}\left(\mathbf{w}_{i}^{\top} \mathbf{a}_{i i}\right)^{2}}
$$

One can prove that the interference function $\mathbf{J}(\mathbf{p})$ whose $i^{\text {th }}$ component is given by (22) is also standard [1] and thus the power control iteration of the form of (10) converges to the optimum power vector. The implementation of the iterative power control algorithm for user $i$ first finds the MMSE $\mathbf{c}_{i}, \mathbf{w}_{i}$ pair for a fixed power vector (since they maximize the SIR and thus are the solution to (22)) and than updates $p_{i}$, i.e. at power control iteration $n+1$ we have

$$
\begin{aligned}
& \left.\left[\hat{\mathbf{c}}_{i}, \hat{\mathbf{w}}_{i}\right]=\arg \min _{\mathbf{c}, \mathbf{w}} E\left[\left(\mathbf{c}^{\top} \mathbf{R}_{i}(\mathbf{p}(n)) \mathbf{w}\right)-b_{i}\right)^{2}\right] \\
& p_{i}(n+1) \\
& =\frac{\gamma_{i}^{*}\left(\sum_{j \neq i} p_{j}(n) h_{i j}\left(\hat{\mathbf{c}}_{i}^{\top} \mathbf{s}_{j}\right)^{2}\left(\hat{\mathbf{w}}_{i}^{\top} \mathbf{a}_{i j}\right)^{2}+\sigma^{2}\left|\hat{\mathbf{c}}_{i}\right|^{2}\left|\hat{\mathbf{w}}_{i}\right|^{2}\right)}{h_{i i}\left(\hat{\mathbf{c}}_{i}^{\top} \mathbf{s}_{i}\right)^{2}\left(\hat{\mathbf{w}}_{i}^{\top} \mathbf{a}_{i j}\right)^{2}}
\end{aligned}
$$

Again, similar to the case in the Power Control and OTSF, if the SIR targets are feasible, then starting from any initial power vector and filter coefficients, the algorithm converges to the minimum power fixed point with best possible temporal-spatial filters in the constrained space. Note that to implement (23) we need to use the iterative algorithm given by (18) and (19).

The filters need to converge to the optimum $\hat{\mathbf{c}}_{i}, \hat{\mathbf{w}}_{i}$ pair for fixed powers before each power update, so, theoretically, many filter updates of the form ((18), (19)) have to be done before power of the user is updated. However, we have observed the convergence of the following algorithm with one of each filter update per power iteration (see Section VII).

$$
\begin{aligned}
\mathbf{c}_{i}(n+1) & =\operatorname{MMSE}\left(\mathbf{w}_{i}(n)\right) \\
\mathbf{w}_{i}(n+1) & =\operatorname{MMSE}\left(\mathbf{c}_{i}(n+1)\right) \\
p_{i}(n+1) & =\frac{\gamma_{i}^{*}}{h_{i i}} \tilde{J}_{i}\left(\mathbf{p}(n), \mathbf{c}_{i}(n+1), \mathbf{w}_{i}(n+1)\right)
\end{aligned}
$$

where

$$
\tilde{J_{i}}\left(\mathbf{p}, \mathbf{c}_{i}, \mathbf{w}_{i}\right)=\frac{\sum_{j \neq i} p_{j} h_{i j}\left(\mathbf{c}_{i}^{\top} \mathbf{s}_{j}\right)^{2}\left(\mathbf{w}_{i}^{\top} \mathbf{a}_{i j}\right)^{2}+\sigma^{2}\left|\mathbf{c}_{i}\right|^{2}\left|\mathbf{w}_{i}\right|^{2}}{\left(\mathbf{c}_{i}^{\top} \mathbf{s}_{i}\right)^{2}\left(\mathbf{w}_{i}^{\top} \mathbf{a}_{i i}\right)^{2}}
$$

\section{Results and Conclusion}

We consider a 9-cell CDMA system with processing gain $G=$ 10. We assume a linear array of omni directional antennas equispaced at half a wavelength [2]. Temporal signatures are generated at random. The SIR target value is the same for all users and is set to $\gamma^{*}=5(7 \mathrm{~dB})$. Results are generated to compare the following algorithms:

1. Conventional power control (C-PC): One antenna and matched filter receiver in time domain [1].

2. Power control and MMSE receiver filter (MMSE-PC): One antenna and SIR maximizing (MMSE) receiver in time domain [6].

3. Power control and beamforming (BF-PC): MMSE combining in spatial domain and matched filter receiver in time domain [7].

4. Power control with $\mathrm{CTSF}\left((\mathrm{c}-\mathrm{w})^{\mathrm{L}}-\mathrm{PC}\right)$ : Constrained temporal-spatial filtering. $\mathrm{L}=5$ iterations of CTSF before each power update.

5. Power control with single step CTSF (c-w-PC): Constrained temporal-spatial filtering, $\mathrm{L}=1$.

6. OTSF (X-PC): Joint unconstrained filtering in temporal and spatial domains.

Figure 1 shows the comparison of total transmit power usage when there are $N=12$ users in the system. An antenna array of $K=2$ elements is used. For this small system, all power control algorithms are feasible, i.e. all users can achieve $\gamma^{*}$. However, we see that the joint spatial temporal algorithms (algorithms 4,5,6) offer savings in total transmit power over the $\mathrm{C}-\mathrm{PC}$ and the combined power control and MMSE filtering in one domain (algorithms 2,3). Compared to C-PC, the savings are as high as $7.2 \mathrm{~dB}$. 
Next, we consider a highly loaded system with $N=60$. The number of antenna array elements is $K=4$. In Figure 2, we see that only power control algorithms with joint processing in both domains are feasible (algorithms 4, 5,6). The system can support this many users only by utilizing the structure in both temporal and spatial domains in conjunction with power control.

Figure 3 emphasizes the fact that the OTSF with power control offers more savings in total transmit power as compared to CTSF with power control (see Section V). The system has $N=60$ users with $K=2$ and only algorithms 4,5 and 6 are feasible. The figure also emphasizes our observation about the convergence of the $L=1$ algorithm implemented as in (25)(28) to the optimal power vector with optimal CTSFs.

In this paper, we have shown that when antenna arrays are employed at each base station, the system performance can be improved by jointly combining the array observations and the temporal observations and employing power control. The total transmit power expended by all users is less as compared to algorithms that do not utilize both temporal and spatial domains. In cases where other algorithms result in an infeasible system, power control with multiuser detection and beamforming can convert the system into a feasible one. Thus, it increases the system capacity by allowing the SIR targets of the users to be higher, or by increasing the number of users supportable at a fixed SIR target level.

\section{References}

[1] R. D. Yates. A framework for uplink power control in cellular radio systems. IEEE JSAC, 13(7):1341-1347, 1995.

[2] S.Verdú. Multiuser Detection. Cambridge University Press, 1998.

[3] A. F. Naguib, A. Paulraj, and T. Kailath. Capacity improvement with base-station antenna arrays in cellular CDMA. IEEE Trans. Veh. Tech., 43(3):691-698, 1994.

[4] S. Verdú. Computational complexity of multiuser detection. Algorithmica, 4(4):303-312, 1989.

[5] U. Madhow and M. L. Honig. MMSE interference suppression for direct-sequence spread-spectrum CDMA. IEEE Trans. Comm., 42(12):3178-3188, 1994.

[6] S. Ulukus and R. D. Yates. Adaptive power control and MMSE interference suppression. ACM Wireless Networks, 4(6):489-496, 1998.

[7] F. Rashid-Farrokhi, L. Tassiulas, and K. J. R. Liu. Joint power control and beamforming in wireless networks using antenna arrays. IEEE Trans. Comm., 46(10):247-256, 1998.

[8] V. G. Subramanian and U. Madhow. Blind demodulation of direct-sequence CDMA signals using an antenna array. In CISS'96.

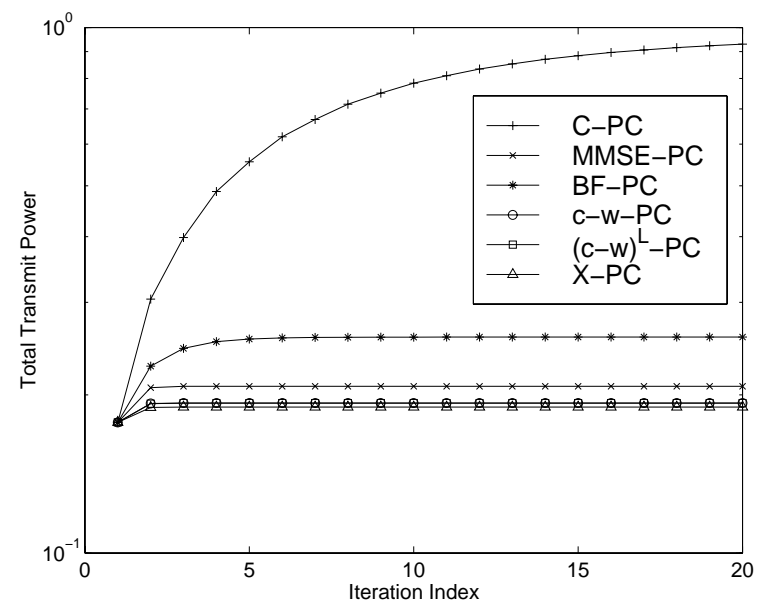

Figure 1: $\mathrm{N}=12, \mathrm{~K}=2$

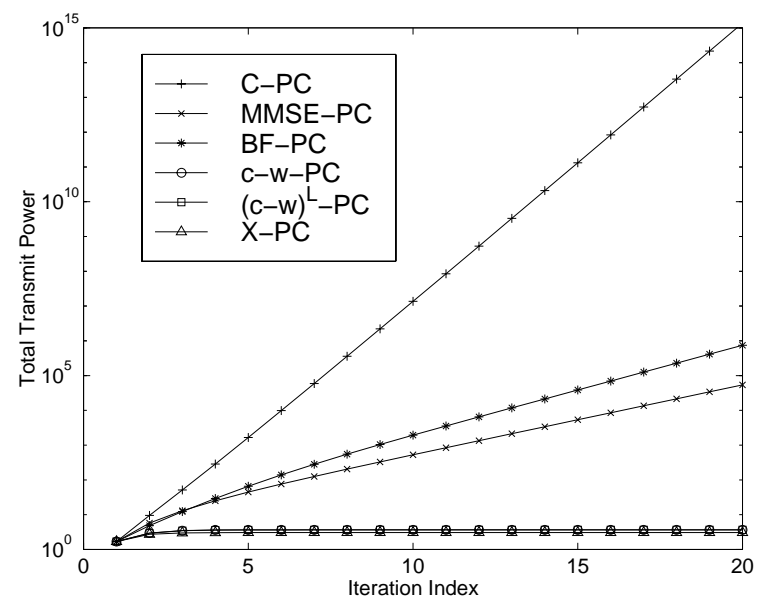

Figure 2: $N=60, K=4$

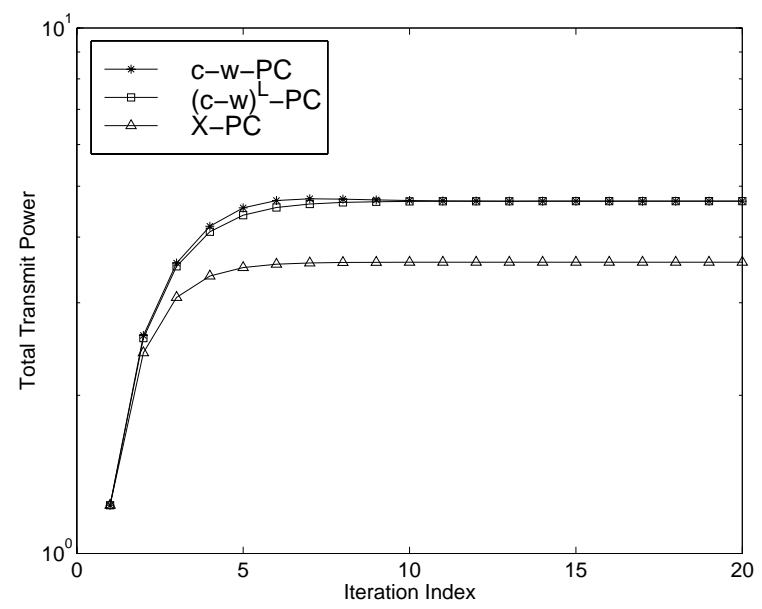

Figure 3: $\mathrm{N}=60, \mathrm{~K}=2$ 